\title{
Significance of Sufficient Neck Flexion During Magnetic Resonance Imaging in the Diagnosis of Hirayama Disease: Report of Two Cases
}

\author{
Seung-Wook No, MD ${ }^{1}$, Duk Hyun Sung, MD, PhD², Du Hwan Kim, MD, PhD ${ }^{1}$
}

\author{
${ }^{1}$ Department of Rehabilitation Medicine, Dongsan Medical Center, Keimyung University School of Medicine, Daegu; \\ ${ }^{2}$ Department of Physical and Rehabilitation Medicine, Samsung Medical Center, \\ Sungkyunkwan University School of Medicine, Seoul, Korea
}

It is difficult to distinguish Hirayama disease (HD) from other mimicking disorders in adolescent patients with distal upper limb weakness. The prevailing theory of HD postulates that the lower cervical cord is susceptible to compression during neck flexion because of insufficient growth of the dura relative to the spinal column. Confirmation of a dynamic change in the dorsal epidural space on magnetic resonance imaging (MRI) during neck flexion is essential for diagnosing HD. However, neck flexion MRI has not been routinely performed in juvenile patients with distal upper limb weakness in the absence of suspected HD. We report two cases of HD that were initially confused with other diseases because of insufficient or absent cervical flexion during MRI. Fullflexion MRI showed typical findings of HD in both cases. Our cases suggest that dynamic cervical MRI in the fully flexed position is necessary for evaluating suspected HD.

Keywords Monomelic amyotrophy, Myelopathy, Magnetic resonance imaging

\section{INTRODUCTION}

Hirayama disease (HD) is a dynamic compressive myelopathy that usually unilaterally affects the distal upper limb, predominantly in young men; it has also been suggested to be an intrinsic motor neuron disease [1,2]. HD is caused by a developmental imbalance between the vertebral column and the spinal cord or dura during the pubertal growth spurt.

Clinical features of HD include bilateral weakness, proximal weakness, or positive upper motor neuron signs $[3,4]$. These atypical manifestations may confuse the diagnosis. For example, spinal neoplasm, lower cervical radiculopathy, distal spinal muscular atrophy, painless neuralgic amyotrophy, neurogenic thoracic outlet syndrome, multifocal motor neuropathy, or amyotrophic

Received October 29, 2018; Accepted November 19, 2018

Corresponding author: Du Hwan Kim

Department of Rehabilitation Medicine, Dongsan Medical Center, Keimyung University School of Medicine, 56 Dalseong-ro, Jung-gu, Daegu 41931, Korea. Tel: +82-53-250-7477, Fax: +82-53-250-7205, E-mail: ripheonix@dsmc.or.kr

ORCID: Seung-Wook No (http://orcid.org/0000-0003-4558-7450); Duk Hyun Sung (http://orcid.org/0000-0002-8261-7199); Du Hwan Kim (http:// orcid.org/0000-0002-9980-8549).

(c) This is an open-access article distributed under the terms of the Creative Commons Attribution Non-Commercial License (http://creativecommons.org/ licenses/by-nc/4.0) which permits unrestricted noncommercial use, distribution, and reproduction in any medium, provided the original work is properly cited. Copyright $\odot 2019$ by Korean Academy of Rehabilitation Medicine 
lateral sclerosis can mimic HD [3,4]. Because HD may be related to dynamic changes in the spinal cord during neck flexion, its diagnosis may be missed or delayed in case of absent or insufficient flexion during cervical magnetic resonance imaging (MRI).

We report two cases of HD that were initially confused with other diseases owing to a suboptimal position of the neck or absence of cervical flexion during MRI. Patients provided written informed consent.

\section{CASE REPORTS}

\section{Case 1}

An 18-year-old male presented with a 5-year history of right-hand weakness with hand muscle atrophy. The weakness progressed for the first 2 years, especially in wrist and finger extension. Posterior interosseous nerve syndrome (PINS) was diagnosed at another hospital. He underwent exploratory surgery 3 years after symptom onset; however, his weakness did not change. Three months before admission to our clinic, he visited another hospital for further evaluation. Because of a high suspicion for HD, routine cervical MRI in the neck-flexion position was performed but did not reveal cord atrophy or dynamic spinal cord compression (Fig. 1A-1D). Considering that cervical MRI showed a negative finding and the weakness manifested as multifocal patchy paresis in the median and ulnar nerve territories, upper-arm MRI was performed, revealing hyperintensity and swelling of the median and ulnar nerves (Fig. 2). Thus, neuralgic amyotrophy was tentatively diagnosed at that hospital. He visited our clinic for a second opinion. On physical examination, wrist and finger flexion, thumb opposition, and finger extension were apparently weak, with $3 / 5$ or

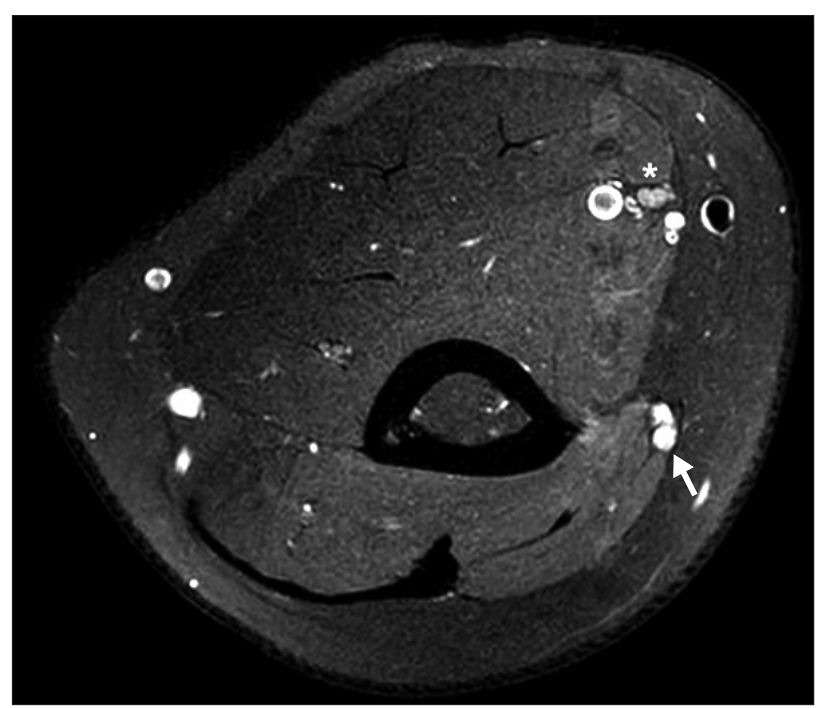

Fig. 2. Upper-arm magnetic resonance imaging revealed hyperintensity and swelling of the median (asterisk) and ulnar (arrow) nerves in the axial T2-weighted fat-suppressed turbo spin-echo sequence image.
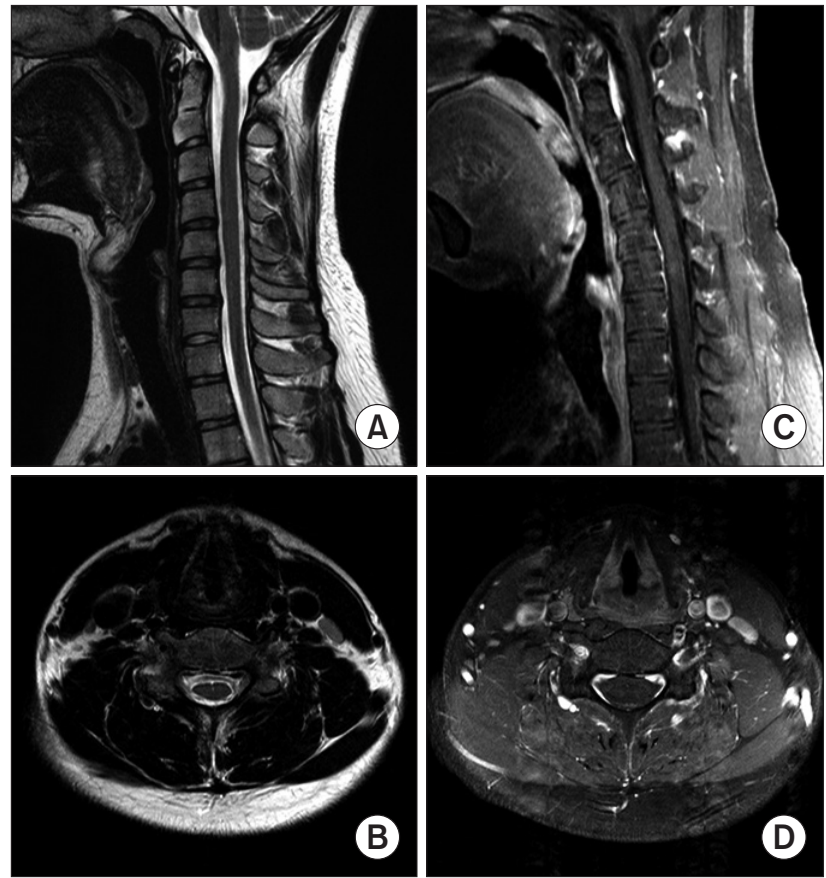
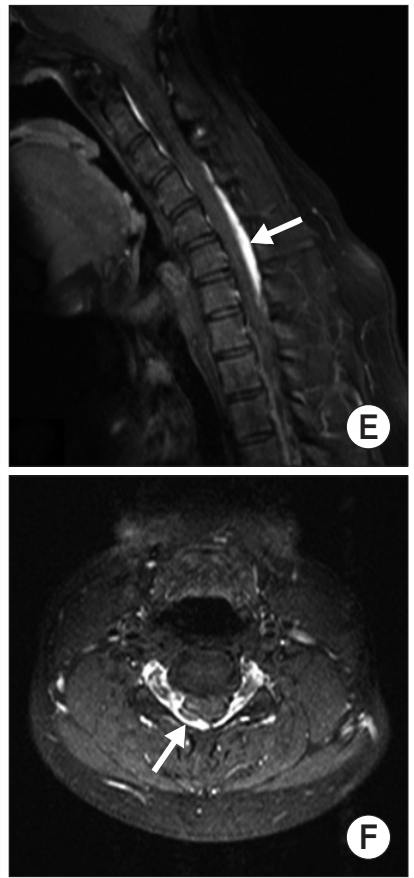

Fig. 1. (A, B) T2-weighted cervical magnetic resonance imaging (MRI) in the neutral position revealed no signal change or spinal cord atrophy. (C, D) Contrastenhanced MRI in the suboptimal neck-flexion position did not show dural detachment from the adjacent lamina. (E) Sagittal contrast-enhanced MRI in the fully flexed position demonstrated a crescent-shaped enlarged posterior epidural space (arrow). (F) Axial contrast-enhanced MRI in the fully flexed position revealed right dural sac detachment from the lamina and spinal cord compression by the engorged posterior epidural venous plexus (arrow). 
Table 1. Electrodiagnostic study in Case 1

\begin{tabular}{|c|c|c|c|c|c|c|}
\hline & \multicolumn{3}{|c|}{ Right side } & \multicolumn{3}{|c|}{ Left side } \\
\hline & Lat (ms) & Amp & $\mathbf{C V}(\mathrm{m} / \mathrm{s})$ & Lat (ms) & Amp & $\mathrm{CV}(\mathrm{m} / \mathrm{s})$ \\
\hline \multicolumn{7}{|l|}{ Sensory } \\
\hline Median (index finger) & 2.45 & 104.5 & - & 2.40 & 90.4 & - \\
\hline Ulnar (little finger) & 2.00 & 95.3 & - & 2.25 & 76.4 & - \\
\hline Radial (thumb) & 1.70 & 31.4 & - & 1.70 & 32.9 & - \\
\hline Lateral antebrachial (forearm) & 1.35 & 12.7 & - & 1.45 & 45.5 & - \\
\hline \multicolumn{7}{|l|}{ Motor } \\
\hline Median (APB) & 3.65 & 11.6 & 59.0 & 3.25 & 14.4 & 61.8 \\
\hline Ulnar (ADM) & 3.40 & 5.3 & 49.4 & 2.60 & 13.0 & 56.0 \\
\hline \multirow[t]{3}{*}{ Radial (EIP) } & 2.15 & 3.3 & - & 2.00 & 7.9 & - \\
\hline & \multicolumn{2}{|c|}{ Spontaneous activity } & \multicolumn{4}{|c|}{ Motor unit action potential } \\
\hline & IA & Fib & PSW & Poly & Duration & $\begin{array}{c}\text { Recruit. } \\
\text { pattern }\end{array}$ \\
\hline \multicolumn{7}{|l|}{ Needle EMG } \\
\hline Right deltoid & $\mathrm{N}$ & - & - & - & $\mathrm{N}$ & $\mathrm{N}$ \\
\hline Right triceps & $\mathrm{N}$ & - & - & - & $\mathrm{N}$ & $\mathrm{N}$ \\
\hline Right biceps & $\mathrm{N}$ & - & - & - & $\mathrm{N}$ & $\mathrm{N}$ \\
\hline Right FCR & $\mathrm{N}$ & - & - & - & $\mathrm{N}$ & $\mathrm{N}$ \\
\hline Right FCU & Increased & +1 & +1 & + & $\mathrm{N}$ & Reduced \\
\hline Right EDC & Increased & +1 & +1 & + & $\mathrm{N}$ & Reduced \\
\hline Right APB & Increased & +1 & +1 & + & $\mathrm{N}$ & $\mathrm{N}$ \\
\hline Right FDI & Increased & +1 & +1 & + & $\mathrm{N}$ & Reduced \\
\hline Right cervical paraspinalis & $\mathrm{N}$ & - & - & - & $\mathrm{N}$ & $\mathrm{N}$ \\
\hline
\end{tabular}

Amplitudes are measured in millivolt ( $\mathrm{mV}$, motor) and in microvolt $(\mu \mathrm{V}$, sensory).

$\mathrm{CV}$, conduction velocity; $\mathrm{APB}$, abductor pollicis brevis; ADM, abductor digiti minimi; EIP, extensor incidis propirus; EMG, electromyography; IA, insertional activity; Fib, fibrillation potential; PSW, positive sharp wave; Poly, polyphasic motor unit; FCR, flexor carpi radialis; FCU, flexor carpi ulnaris; EDC, extensor digitorum communis; FDI, first dorsal interosseous; $\mathrm{N}$, normal.

4/5 strength on the Medical Research Council (MRC) scale. There were no fasciculations or sensory deficits. Sensory nerve conduction study (NCS) results were normal (Table 1). Motor NCSs on the ulnar and radial nerves showed a marked amplitude decrement relative to the unaffected side (Table 1). Needle electromyography revealed denervation potentials and polyphasic motor unit action potentials in C7-T1 innervated muscles (Table 1). Considering the electrodiagnostic results, we suspected HD rather than PINS or neuralgic amyotrophy. Accordingly, we repeated contrast-enhanced cervical MRI with sufficient flexion, which showed dural sac detachment from the lamina and spinal cord compression by the engorged posterior epidural venous plexus (Fig. 1E, 1F).

\section{Case 2}

A 16-year-old male with a 3-month history of progressive right-wrist extensor weakness visited our hospital. Cervical MRI showed disc protrusion at C5-6 and C6-7. We suspected that these findings were related to symptoms (Fig. 3A). However, another hand surgeon clinically diagnosed the condition as PINS. At the first visit, an electrodiagnostic study revealed abnormal findings, including decreased amplitudes in the right radial nerve and denervation potentials in the right extensor digitorum communis and extensor indicis proprius, suggesting PINS rather than lower cervical radiculopathy (Table 2). There were no abnormal findings of somatosensory-evoked potentials on both upper and lower limbs. However, his weakness progressed to intrinsic hand muscle involve- 

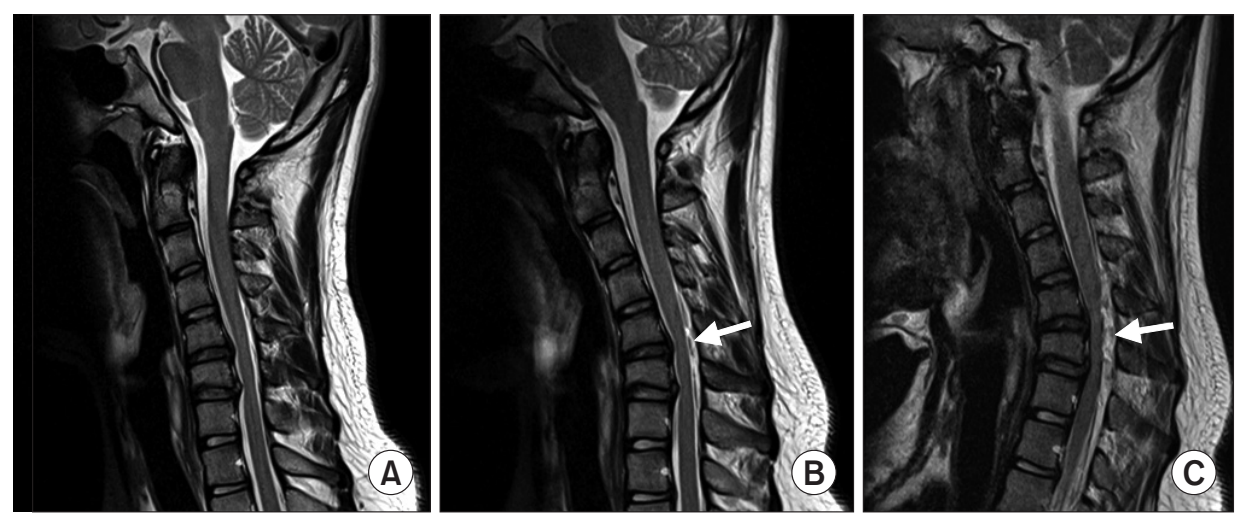

Fig. 3. (A) Cervical magnetic resonance imaging (MRI) in the neutral position showed disc protrusion at C5-6 and C6-7 in the sagittal T2-weighted image. (B) Cervical MRI with neck flexion revealed suspected dural detachment from the lamina (arrow). (C) Cervical MRI with full flexion demonstrated obvious widening of the dorsal epidural space with flattening of the cervical cord (arrow).

ment over 3 weeks. Subtle atrophic changes of the righthand intrinsic muscles and forearm muscles were noted. Physical examination showed right-sided weakness of wrist flexion, finger flexion, and thumb opposition with MRC 4/5 strength. Signs of sensory deficit or root irritation were absent. Follow-up needle electromyography revealed denervation potentials in right ulnar territory muscles (Table 2). Considering the follow-up electrodiagnostic study results and clinical manifestations, we suspected HD rather than cervical radiculopathy or PINS. Cervical MRI was repeated with neck flexion. Dural sac detachment from the lamina was suspected (Fig. 3B). Contrast-enhanced MRI in a fully flexed position showed an obvious anterior shift of the lower cervical cord and widening of the dorsal epidural space (Fig. 3C).

\section{DISCUSSION}

We described cervical MRI findings with sufficient neck flexion in comparison with MRI in the neutral or insufficient-flexion position in 2 patients with HD. The neutral position or insufficient flexion during MRI resulted in misdiagnosis (as PINS, cervical radiculopathy, or neuralgic amyotrophy). In contrast, MRI with adequate flexion showed typical findings of HD (posterior dural detachment with an anterior shift causing focal cord compression and/or crescent-shaped enlarged posterior epidural space with engorged epidural veins).

It is often difficult to differentiate HD from other mimicking diseases, including lower cervical radiculopathy, distal spinal muscular atrophy, painless neuralgic amyotrophy, and multifocal motor neuropathy. With clinical manifestations or an electrodiagnostic study alone, delayed diagnosis or misdiagnosis may occur. Even patients with typical HD might show negative MRI findings (frequency, 24\%-29\%) [5,6]. Our report suggests that additional full-flexion MRI is helpful when conventional neck-flexion MRI is negative.

Hirayama et al. [7] reported on 12 patients with juvenile muscular atrophy of the distal upper extremity in 1959. Hirayama et al. [3] reported cord flattening at C7 and C8. Cervical MRI in dynamic positions may be a key step in diagnosing HD. Hou et al. reported that anterior shifting of the posterior wall was significantly affected by the neck-flexion angle in patients with HD [8]. However, there is no consensus on the optimum condition of neck flexion during cervical MRI in diagnosing HD, and neckflexion MRI is not routinely performed in juvenile patients with distal upper-limb weakness in the absence of suspected HD or if neutral cervical MRI does not reveal cord atrophy.

HD pathophysiology remains unclear. However, a developmental imbalance between the vertebral column and the spinal cord or dural canal during the pubertal growth spurt is known to be associated with HD. The dural canal becomes tight during neck flexion, causing an anterior shift of the posterior dural wall and spinal cord. This anterior shift leads to cervical cord compression, causing microcirculatory disturbances in the anterior horn of the lower cervical spinal cord [9]. Chronic circu- 
Table 2. A summary of initial and follow-up electrodiagnostic study in Case 2

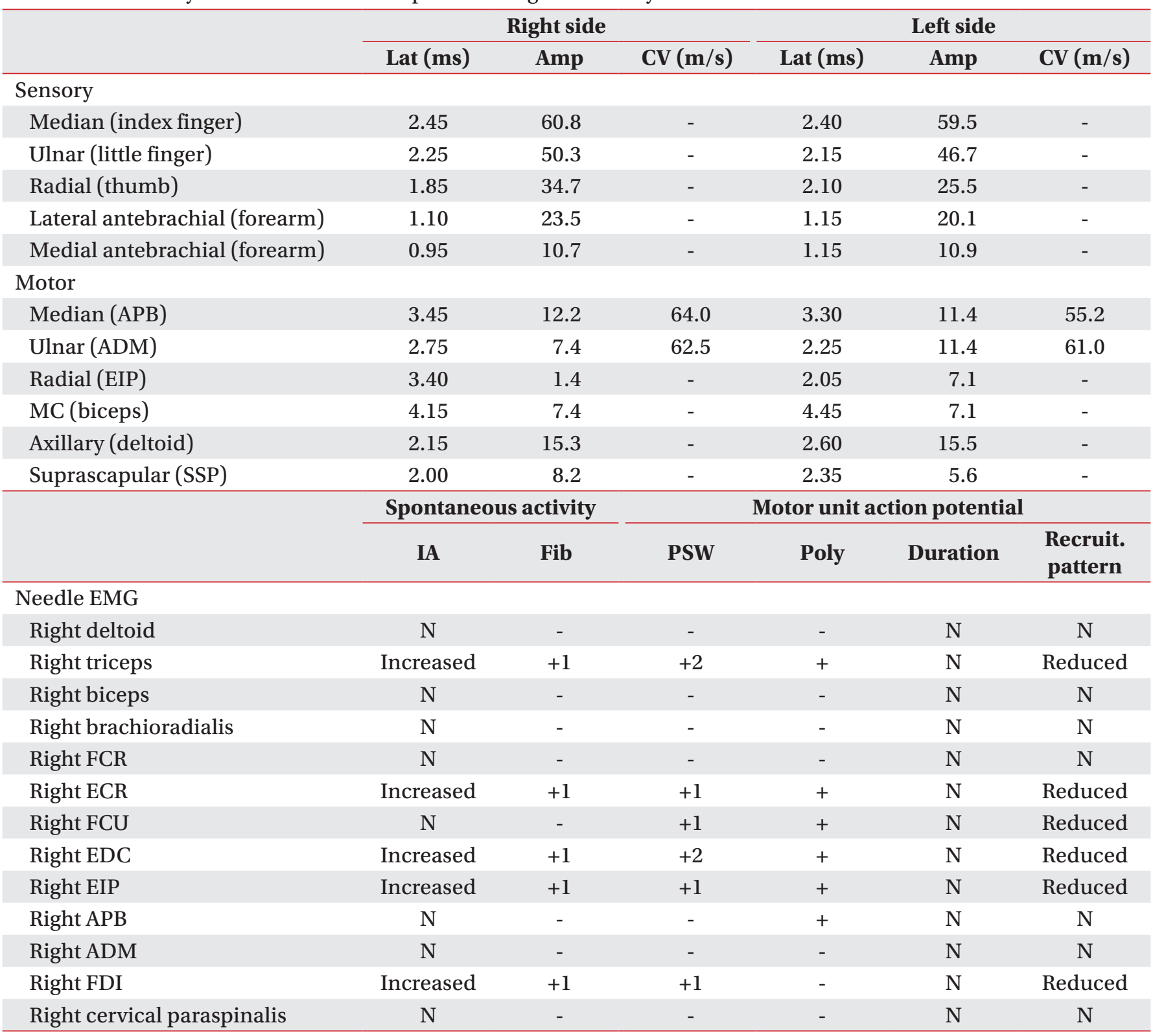

Amplitudes are measured in millivolt ( $\mathrm{mV}$, motor) and in microvolt ( $\mu \mathrm{V}$, sensory).

$\mathrm{CV}$, conduction velocity; APB, abductor pollicis brevis; ADM, abductor digiti minimi; EIP, extensor incidis propirus; EMG, electromyography; IA, insertional activity; Fib, fibrillation potential; PSW, positive sharp wave; Poly, polyphasic motor unit; FCR, flexor carpi radialis; ECR, extensor carpi radialis; FCU, flexor carpi ulnaris; EDC, extensor digitorum communis; EIP, extensor incidis propirus; APB, abductor pollicis brevis; ADM, abductor digiti minimi; FDI, first dorsal interosseous; $\mathrm{N}$, normal.

latory disturbances resulting from repeated or sustained neck flexion may cause degeneration of anterior horn cells, which are highly susceptible to ischemia [10].

Case 1 showed structural changes in peripheral nerves on 3-T upper-arm MRI. Swelling and signal changes in the median and ulnar nerves are interesting and unique clinical findings of lower cervical cord compression. Microscopically, neural reaction to injury occurs in the nerve portion distal to the lesion (referred to as Wallerian degeneration). Anterior horn cell degeneration due to dynamic compression or secondary ischemic damage may cause Wallerian degeneration, which might be visu- 
alized in limb MRI. To our knowledge, the visualization of structural changes of peripheral nerves caused by dynamic spinal cord compression has not been reported.

In conclusion, cervical MRI in dynamic positions, including full-flexion MRI, is recommended in juvenile patients whose conventional cervical MRI revealed negative findings despite typical clinical manifestations and evidence of focal motor neuron disease in electrodiagnostic studies.

\section{CONFLICT OF INTEREST}

No potential conflict of interest relevant to this article was reported.

\section{AUTHOR CONTRIBUTION}

Conceptualization: Kim DH, Sung DH. Methodology: Sung DH. Formal analysis: Kim DH, Sung DH. Project administration: Kim DH, No SW. Visualization: Kim DH, No SW. Writing - original draft: Kim DH, No SW. Writing - review and editing: Sung DH. Approval of final manuscript: all authors.

\section{REFERENCES}

1. Schroder R, Keller E, Flacke S, Schmidt S, Pohl C, Klockgether T, et al. MRI findings in Hirayama's disease: flexion-induced cervical myelopathy or intrinsic motor neuron disease? J Neurol 1999;246:1069-74.

2. Tashiro K, Kikuchi S, Itoyama Y, Tokumaru Y, Sobue
G, Mukai E, et al. Nationwide survey of juvenile muscular atrophy of distal upper extremity (Hirayama disease) in Japan. Amyotroph Lateral Scler 2006;7:38-45.

3. Hirayama K. Juvenile muscular atrophy of distal upper extremity (Hirayama disease). Intern Med 2000;39: 283-90.

4. Yoo SD, Kim HS, Yun DH, Kim DH, Chon J, Lee SA, et al. Monomelic amyotrophy (Hirayama disease) with upper motor neuron signs: a case report. Ann Rehabil Med 2015;39:122-7.

5. Lehman VT, Luetmer PH, Sorenson EJ, Carter RE, Gupta V, Fletcher GP, et al. Cervical spine MR imaging findings of patients with Hirayama disease in North America: a multisite study. AJNR Am J Neuroradiol 2013;34:451-6.

6. Zhou B, Chen L, Fan D, Zhou D. Clinical features of Hirayama disease in mainland China. Amyotroph Lateral Scler 2010;11:133-9.

7. Hirayama K, Toyokura Y, Tsubaki T. Juvenile muscular atrophy of unilateral upper extremity: a new clinical entity. Psychiatr Neurol Jpn 1959;61:2190-8.

8. Hou C, Han H, Yang X, Xu X, Gao H, Fan D, et al. How does the neck flexion affect the cervical MRI features of Hirayama disease? Neurol Sci 2012;33:1101-5.

9. Hirayama K, Tokumaru Y. Cervical dural sac and spinal cord in juvenile muscular atrophy of distal upper extremity. Neurology 2000;54:1922-6.

10. Lapresle J. Clinical and neuropathological aspects of circulatory disorders of the spinal cord. Bull Schweiz Akad Med Wiss 1969;24:512-28. 